\title{
In vivo noninvasive monitoring of microhemodynamics using optical- resolution photoacoustic microscopy
}

Song Hu, Konstantin I. Maslov, Lihong V. Wang

Song Hu, Konstantin I. Maslov, Lihong V. Wang, "In vivo noninvasive monitoring of microhemodynamics using optical-resolution photoacoustic microscopy," Proc. SPIE 7177, Photons Plus Ultrasound: Imaging and Sensing 2009, 71770H (24 February 2009); doi: 10.1117/12.809297 


\title{
In Vivo Noninvasive Monitoring of Microhemodynamics Using Optical-Resolution Photoacoustic Microscopy
}

\author{
Song $\mathrm{Hu}$, Konstantin I. Maslov, and Lihong V. Wang \\ Optical Imaging Laboratory, Department of Biomedical Engineering, \\ Washington University in St. Louis, St. Louis, Missouri, USA 63130
}

\begin{abstract}
Microvascular autoregulation is an intrinsic ability of vascular beds to compensate for the fluctuation in blood flow and tissue oxygen delivery. This function is crucial to maintaining the local metabolic activity. Here, using optical-resolution photoacoustic microscopy (OR-PAM), we clearly observed vasomotion and vasodilation in the intact mouse microcirculation in vivo in response to the changes in physiological state. Our results show that a significant lowfrequency vasomotion can be seen under hyperoxia but not hypoxia. Moreover, significant vasodilation is observed when the animal status is switched from hyperoxia to hypoxia. Our data show that arterioles have more pronounced vasodilation than venules.
\end{abstract}

Keywords: Optical-resolution photoacoustic microscopy, microvascular autoregulation, vasomotion, vasodilation.

\section{INTRODUCTION}

Microcirculation is the terminal functional unit of the cardiovascular system, carrying out the exchange of gas and nutrients between blood and tissue. In vivo noninvasive microvascular imaging is of great physiological and clinical importance. However, until now, there has not been a technique that could provide comprehensive and accurate information of the intact microcirculation.

To fill this gap, we have developed optical-resolution photoacoustic microscopy (OR-PAM) ${ }^{1,2}$. Benefiting from the physiologically specific endogenous optical absorption contrast, OR-PAM enables in vivo noninvasive contrast-agentfree three-dimensional microvascular imaging down to the capillary level.

In this work, we monitored vasomotion and vasodilation - two important microhemodynamic activities in tissue oxygen regulation - in the intact microcirculation in small animals in vivo. The good agreements between our observations and the previous invasive studies validate the feasibility of OR-PAM. The noninvasiveness and label-free feature of ORPAM enables a more accurate and objective view on the microcirculation.

\section{METHODS AND MATERIALS}

\subsection{OR-PAM with diffraction-limited optical focusing}

As shown in Fig. 1(A), in our OR-PAM system, a wavelength-tunable laser set, consisting of a dye laser an Nd:YLF pump laser, is used as the excitation source. Laser beam coming out of the dye laser is attenuated and spatially filtered by a pinhole. The reshaped laser beam is then focused into a diffraction-limited optical focal spot by an objective lens. Ultrasonic focusing is achieved through a plano-concave attached under the right-angle prism. The optical objective lens and the ultrasonic transducer are configured coaxially and confocally.

Photons Plus Ultrasound: Imaging and Sensing 2009, edited by Alexander A. Oraevsky, Lihong V. Wang, Proc. of SPIE Vol. 7177, 71770H · @ 2009 SPIE · CCC code: 1605-7422/09/\$18 · doi: 10.1117/12.809297 
In this acoustic-optical confocal configuration, the lateral resolution is determined by the product of the two pointspread-functions of optical illumination and acoustic detection. Here, we use diffraction-limited optical focusing to achieve the capillary-level lateral resolution. As shown in Fig. 1(B), within the 27- $\mu$ m-diameter acoustic focus, only a 5$\mu \mathrm{m}$-diameter spot is optically excited, so the detected photoacoustic signal is exclusively generated within the optical focus. Thereby, the lateral resolution of photoacoustic microscopy can be easily promoted from $50 \mu \mathrm{m}^{3-5}$ to $5 \mu \mathrm{m}$ or even higher.
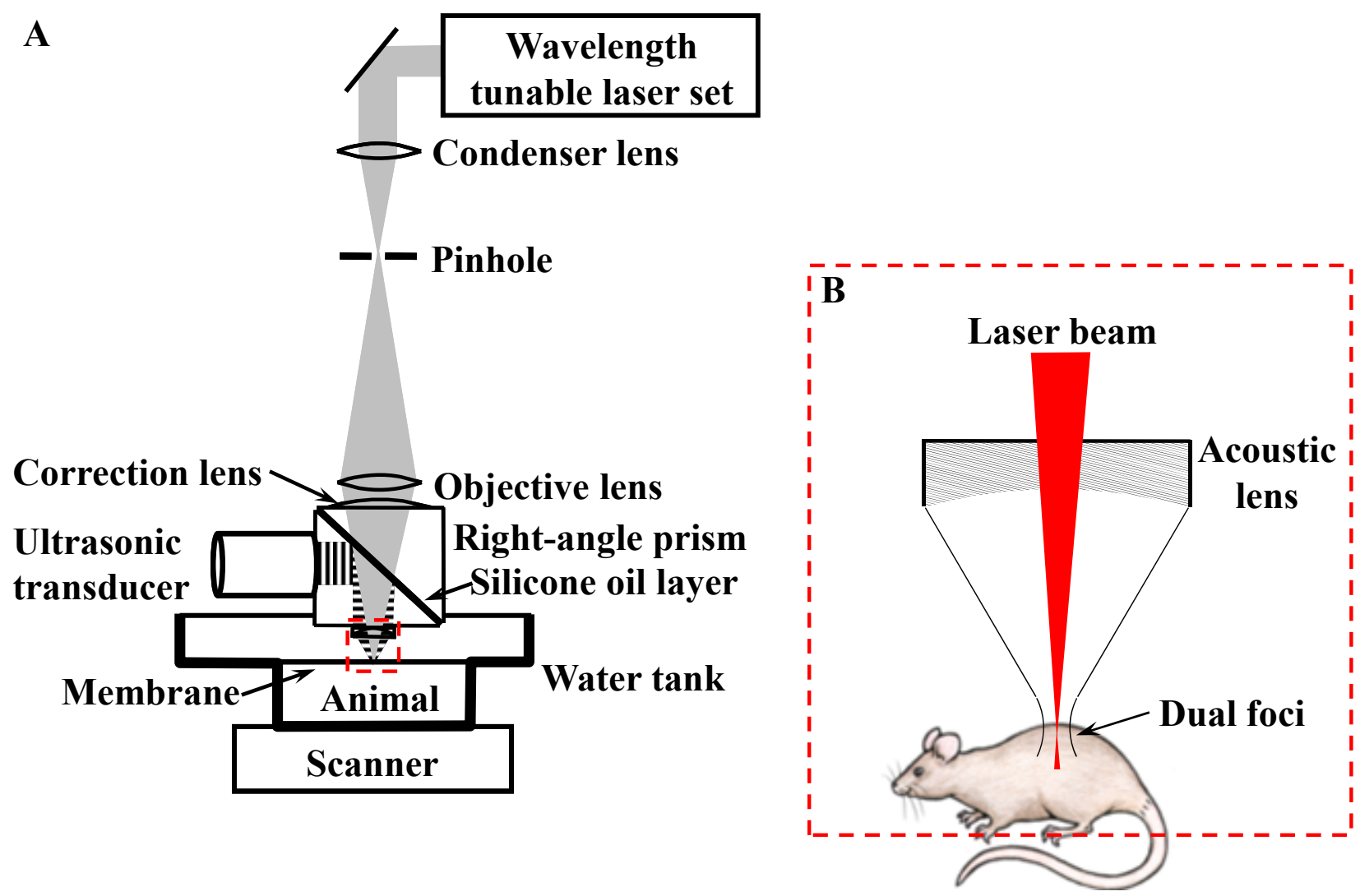

Figure 1. (A) Schematic of the OR-PAM system ${ }^{1,2}$. (B) A blow up sketch of the diffraction-limited optical illumination and the acoustic-optical confocal configuration.

\subsection{Animal preparation}

All experimental animal procedures were carried out in conformance with the laboratory animal protocol approved by the School of Medicine Animal Studies Committee of Washington University in St. Louis.

Nude mice (Hsd:Athymic Nude-Foxn 1NU, Harlan Co.) were used. Before imaging, the hair on the ears was gently removed with human hair-removing lotion. A dose of $87 \mathrm{mg} / \mathrm{kg}$ Ketamine and $13 \mathrm{mg} / \mathrm{kg}$ Xylazine was administered intraperitoneally to anesthetize the animal, and anesthesia was maintained throughout the experiments using an isoflurane machine (1.0-1.5\% vaporized isoflurane with an airflow rate of 1 liter/min). The body temperature of the animal was maintained at $37^{\circ} \mathrm{C}$ with a temperature controlled heating pad. At the end of the experiment, the animals were killed by an intraperitoneal administration of pentobarbital at a dosage of $100 \mathrm{mg} / \mathrm{kg}$. 


\section{RESULTS AND DISCUSSION}

\subsection{In vivo microvascular imaging}

A $1 \mathrm{~mm}$-by-1mm region in a nude mouse ear was imaged in vivo noninvasively by OR-PAM at the isosbestic wavelength of $570 \mathrm{~nm}$. The region of interest was scanned with a step size of $2.5 \mu \mathrm{m}$. The scanning time for a complete volumetric dataset was $\sim 6 \mathrm{~min}$. After data acquisition, the animal recovered naturally without observable laser damage.

A
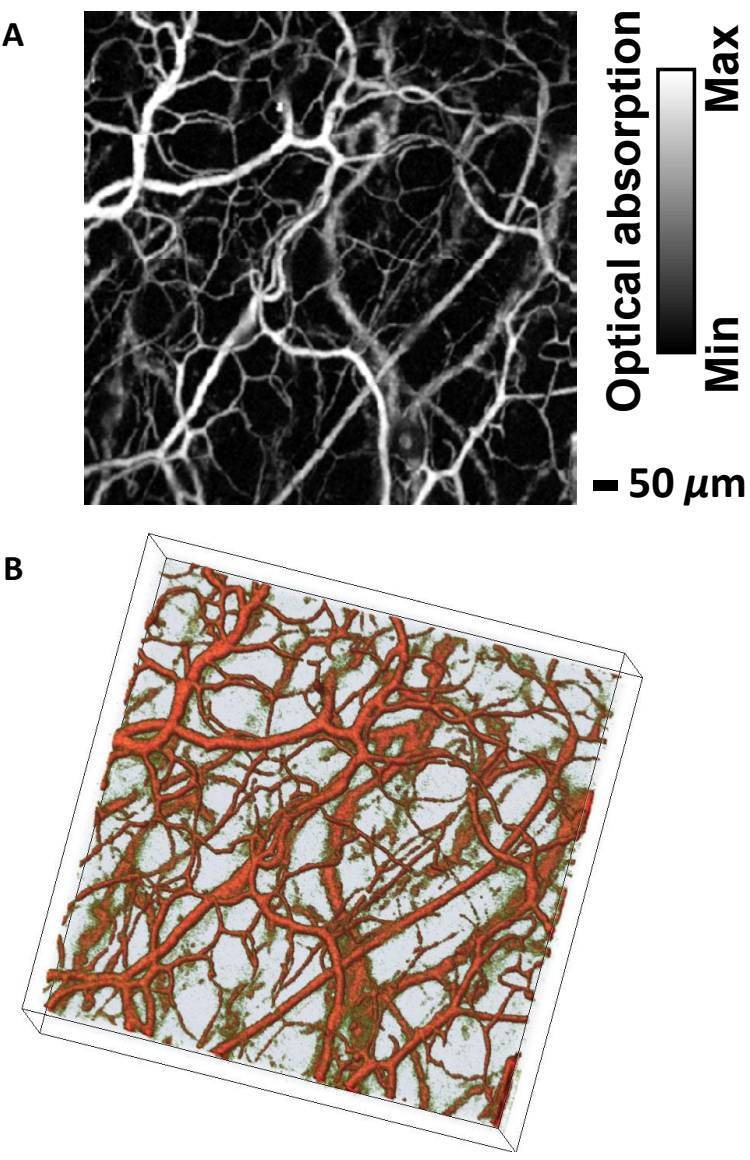

C

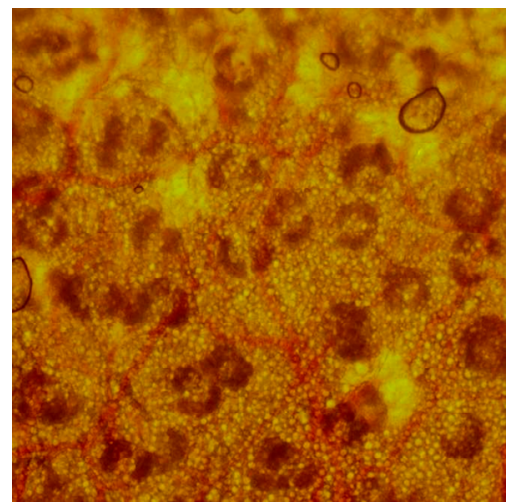

Figure 2. Microvasculature in a nude mouse ear. (A) The maximum-amplitude-projection image acquired in vivo by OR-PAM; (B) 3D pseudocolor visualization of the microvasculature; (C) The photograph taken by a transmission-mode optical microscope. 


\subsection{In vivo monitoring of vasomotion and vasodilation in intact microcirculations}

Vasomotion is the oscillation of vascular tone or vascular diameter that can be generally seen in the cardiovascular system $^{6}$. It is well known not to be a consequence of the heart beat, respiration, or neuronal input; however, its physiological role and its underlying mechanism remain elusive ${ }^{7}$. Vasodilation is better understood to be increase in vessel diameter. Both of them are important vessel activities in tissue oxygen regulation.

To study vasomotion and vasodilation in the intact microcirculation, we randomly imaged a microvascular network in an ear of a living nude mouse, and then selected a representative B-scan crossing several arterioles and venules for monitoring purpose. To induce vasomotion and vasodilation in vivo, we changed the tissue oxygenation of the experimental mouse by alternating the inspired gas between pure oxygen and hypoxic gas $\left(5 \% \mathrm{O}_{2}, 5 \% \mathrm{CO}_{2}\right.$ and $90 \%$ $\mathrm{N}_{2}$ ). We monitored the selected B-scan continuously for seventy minutes during the switching of physiological state, as shown in Fig. 3. Vasomotion under hyperoxia is clearly seen in the form of the oscillation of the vessel cross sections; vasodilation under hypoxia is also clearly observed as the expansion of the vessel diameters.

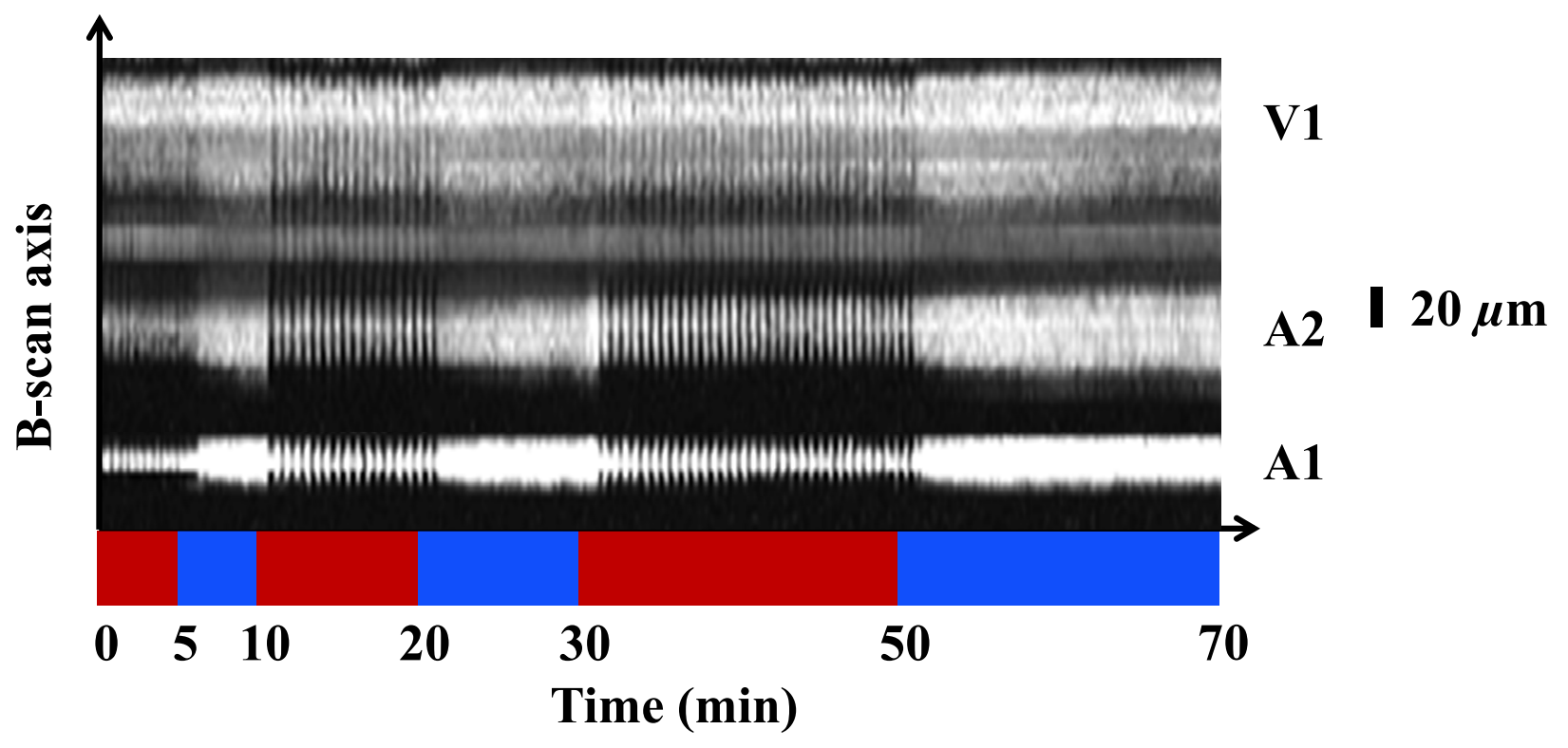

\section{Hyperoxia Hypoxia}

Figure 3. B-scan monitoring of the arteriolar and venular vasomotion and vasodilation in response to the changes in physiological state. A1 and A2 are two selected arterioles; V1 is the selected venule.

Thanks to the high resolution and high sensitivity of OR-PAM measurements, we can study the vasodilation in a more quantitative way by estimating the vessel diameters using the full-width-at-half-maximum values of the vessel cross sectional signals. To eliminate the vessel diameter oscillation due to vasomotion, we employed 60-point-movingaveraging. The smoothed time courses of the arteriolar and venular diameter changes in response to the switching of the physiological state are shown in Fig. 4. It clearly shows that arterioles A1 and A2 have a more significant vasodilation $(80 \%-100 \%)$ than venule V1 (30\%-35\%). 


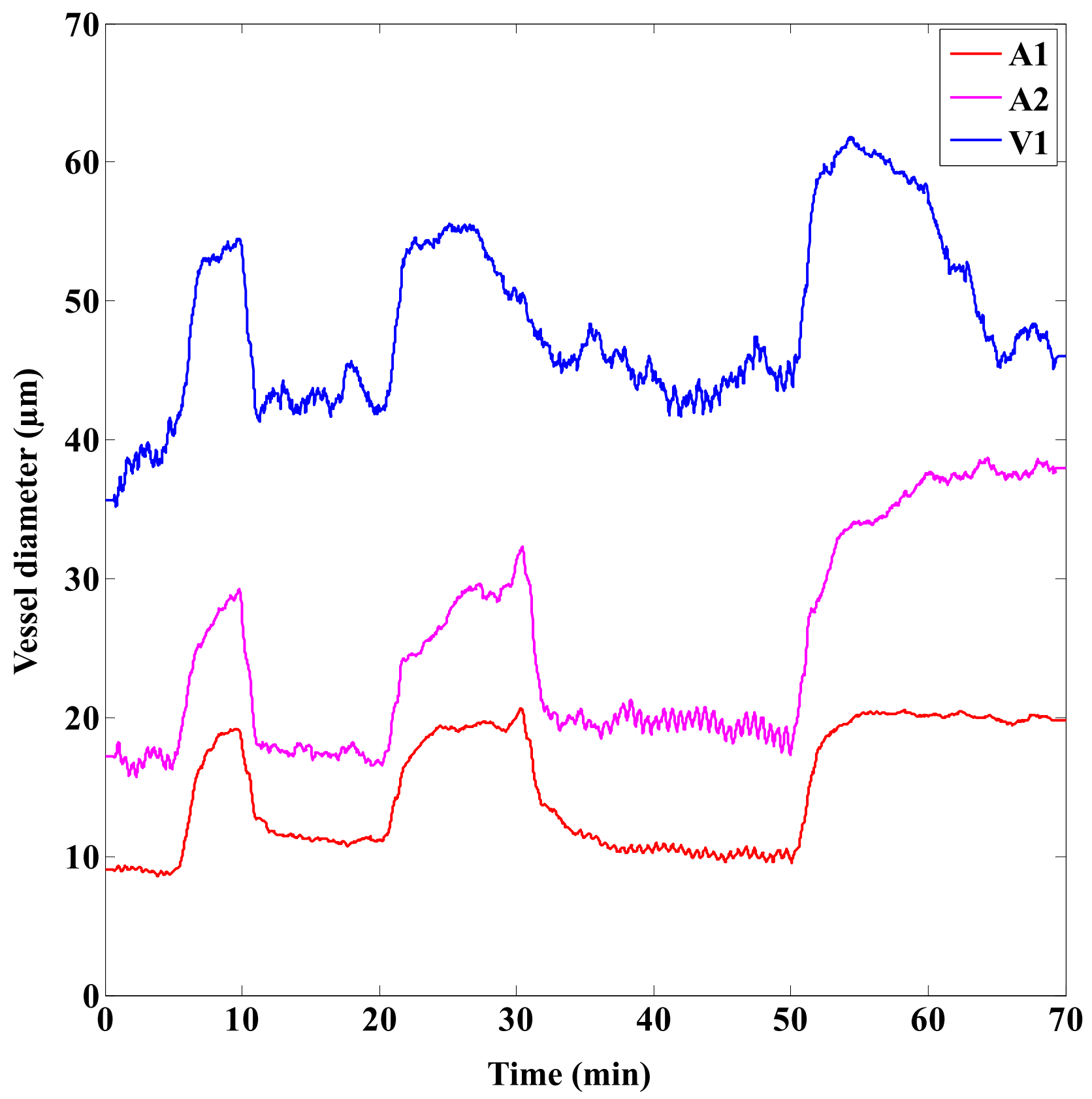

Figure 4. The arteriolar and venular vasodilation in response to hypoxia.

The adequate temporal resolution of OR-PAM also permits quantifying the response time of vasodilation to hypoxia. As shown in Fig. 5, the response time is estimated to be 2-3 minutes. 


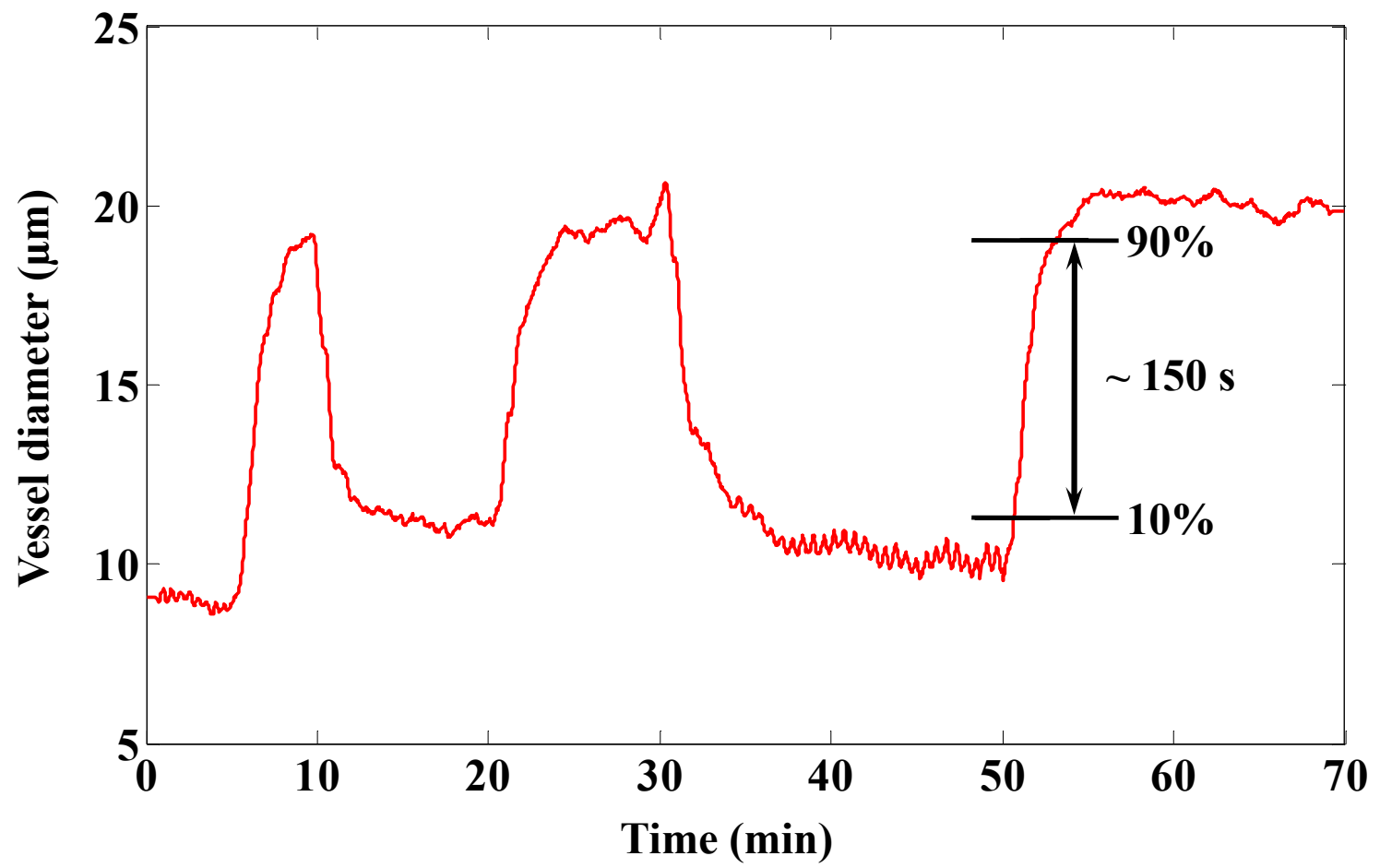

Figure 5. The response time of arteriolar vasodilation to hypoxia.

\section{CONCLUSIONS}

OR-PAM, tracing the physiologically specific absorption signatures of endogenous chromophores, is a valuable complement to the existing scattering- and fluorescence-contrast microscopic techniques. In this work, we successfully demonstrated in vivo monitoring of two important vessel activities - vasomotion and vasodilation - in the intact microcirculation by taking advantage of the endogenous optical absorption contrast of hemoglobin. Our results are in good agreement with the previous invasive studies. OR-PAM provides a more accurate and objective view on the microcirculation by avoiding the possible disturbance due to invasive procedures or exogenous contrast agents.

\section{ACKNOWLEDGEMENTS}

This work was sponsored by National Institutes of Health grants R01 EB000712, R01 NS46214 (Bioengineering Research Partnerships), R01 EB008085, and U54 CA136398 (Network for Translational Research). L.W. has a financial interest in Endra, Inc., which, however, did not support this work. 


\section{REFERENCES}

1. K. Maslov, H.F.Z., S. Hu, and L.V. Wang Optical-resolution confocal photoacoustic microscopy. Proc. SPIE 6856, 68561I (2008).

2. Maslov, K., Zhang, H.F., Hu, S. \& Wang, L.V. Optical-resolution photoacoustic microscopy for in vivo imaging of single capillaries. Opt Lett 33, 929-931 (2008).

3. Maslov, K., Stoica, G. \& Wang, L.V. In vivo dark-field reflection-mode photoacoustic microscopy. Opt Lett 30, 625-627 (2005).

4. Zhang, H.F., Maslov, K., Stoica, G. \& Wang, L.V. Functional photoacoustic microscopy for high-resolution and noninvasive in vivo imaging. Nat Biotechnol 24, 848-851 (2006).

5. Zhang, H.F., Maslov, K. \& Wang, L.V. In vivo imaging of subcutaneous structures using functional photoacoustic microscopy. Nat Protoc 2, 797-804 (2007).

6. Aalkaer, C. \& Nilsson, H. Vasomotion: cellular background for the oscillator and for the synchronization of smooth muscle cells. British journal of pharmacology 144, 605-616 (2005).

7. Nilsson, H. \& Aalkjaer, C. Vasomotion: mechanisms and physiological importance. Molecular interventions 3, 79-89, 51 (2003). 